\title{
The Charcoal Quantification Tool (CharTool): A Suite of Open-source Tools for Quantifying Charcoal Fragments and Sediment Properties in Archaeological and Paleoecological Analysis
}

\author{
Grant Snitker ${ }^{1^{*}}$ \\ ${ }^{1}$ Department of Crop and Soil Sciences, College of Agricultural and Environmental Sciences, University of Georgia, Athens, \\ USA. \\ * grant.snitker@uga.edu
}

\begin{abstract}
Sedimentary charcoal analysis is increasingly used in archaeological and paleoecological research to examine human-environmental relationships at multiple scales. The recent availability of low-cost digital microscopes and imaging software has resulted in the rapid adoption of digital image analysis in charcoal studies. However, most published studies include only minimal accounts of software configurations or utilize proprietary image analysis programs, thus hindering replication, standardization, and comparability of charcoal analyses across the field. In an effort to encourage replicable methods and a culture of open science, this paper presents the Charcoal Quantification Tool (CharTool), a free, open-source suite of charcoal and sediment quantification tools designed for use with ImageJ. CharTool blends standard methods in visual and digital charcoal analysis to increase the analyst's participation in identifying and measuring charcoal metrics. Each CharTool module is described and demonstrated in a vignette using sedimentary charcoal collected from the Son Servera study area, Mallorca, Spain. A suggested workflow, user-guide, scripted analyses for processing outputs, and download instructions are included as supplementary materials to this article.
\end{abstract}

Received October 6, 2019

OPEN ӘACCESS

Accepted June 19, 2020

Published September 18, 2020

DOI 10.14237/ebl.11.1.2020.1653

Keywords Archaeology, Paleoecology, Charcoal Analysis, Image Analysis, ImageJ, R

Copyright $(\subset) 2020$ by the author(s); licensee Society of Ethnobiology. This is an open-access article distributed under the terms of the Creative Commons Attribution-NonCommercial 4.0 International Public License (https://creativecommons.org/licenses/by-nc/4.0), which permits non-commercial use, distribution, and reproduction in any medium, provided the original author and source are credited.

Supplementary Files available at https://doi.org/10.14237/ebl.11.1.2020.1653

\section{Introduction}

Sedimentary charcoal is the primary proxy used in reconstructing past fire activity (Whitlock and Anderson 2003). Charcoal accumulation observed at multiple spatial and temporal scales can provide insights into the bio-climatic drivers of landscape fire and regional vegetation dynamics (Whitlock and Larsen 2001), as well as anthropogenic influences on fire and fuel dynamics through intentional burning (Bowman et al. 2011), fuel wood harvesting practices (Nelle et al. 2013), and other long-term humanenvironmental relationships (Bliege Bird et al. 2018). Visual counts and measurements at low magnification remain the standard method for quantifying charcoal fragment abundance across multiple disciplines (Whitlock and Anderson 2003). In recent years, greater access to digital microscopes and image analysis software has presented new opportunities for the development of digital charcoal quantification procedures (Halsall et al. 2018; Hawthorne and Mitchell 2016; Thevenon and Anselmetti 2007).

Most digital charcoal analysis relies primarily on greyscale video or still images to isolate and quantify optically dense (i.e., dark-colored) charcoal fragments (Halsall et al. 2018). While the functional advantages and disadvantages of automated and digital charcoal analysis have been evaluated in the literature (see Whitlock and Larsen 2001 for an overview), there has been relatively little engagement with the specific challenges (e.g., digital data creation, access, and curation) associated with digital data generated through these methods. To exacerbate these issues, 
often-minimal descriptions of software configurations and the use of paywall-protected and proprietary image analysis software programs prevent replication, standardization, and comparability of charcoal analyses across research projects.

Limitations such as these are the focus of the growing interdisciplinary open science movement in the paleoecological (Williams et al. 2018a), archaeological (Marwick et al. 2017), and paleo-fire science communities (Aleman et al. 2018). Open science seeks to encourage new norms in research that emphasize data stewardship, analytical transparency, and reproducibility through practices of open access, open data, and open methods (Bartling and Friesike 2014; Marwick et al. 2017). In an effort to align digital charcoal analysis with the principles of the open science movement, this paper presents the Charcoal Quantification Tool (CharTool), a suite of charcoal and sediment quantification tools designed for use with ImageJ (Schneider et al. 2012), a free, opensource image and video analysis program. CharTool is not an automated procedure for identifying charcoal fragments. Rather, it is an open and flexible suite of tools for charcoal analysis that blend standard visual protocols (Schlachter and Horn 2010; Whitlock and Anderson 2003) and ImageJ functions common in digital methods (Halsall et al. 2018) to assist an analyst in quantifying charcoal abundance, fragment size, and shape metrics. CharTool also includes an optional workflow for an analyst to digitally record charcoal morphotypes and modules designed to quantify sediment properties, such as sediment color and mean clast size, from cores or excavated contexts. CharTool is freely available ${ }^{1}$, utilizes scripted modules for both transparency and user-customization, and offers a set of standardized tools that can generate comparable datasets across multiple projects.

\section{Previous Methods for Quantifying Charcoal in Archaeological and Paleoecological Research}

Visual and Automated Methods for Quantifying Charcoal Fragments

Traditional methods in microscopy for quantifying charcoal abundance rely on charcoal fragments' visual properties (e.g., opacity, color, planar shape, visual anatomical, and cellular structures) and physical properties (e.g., fragility and breakage pattern) to make them distinguishable from minerals, insect exoskeletons, or other plant tissues under magnification (Scott 2010). Common procedures consist of visually or physically isolating charcoal fragments from sampled sediments and quantifying abundance by a count, area, or mass (Schlachter and Horn 2010; Whitlock and Larsen 2001). Quantifying additional charcoal attributes, such as size class or fragment length, is accomplished using a stereoscope fitted with a measure scale (Ali et al. 2009).

In the early 1990s, Horn et al. (1992) proposed automated digital image analysis as an avenue for improving the speed and replicability of quantifying charcoal abundance, including measures of both charcoal count and area. Early applications of automated analysis were limited, as they required specialized microscope-mounted cameras with computer connectivity, proprietary imaging software, and multiple calibration trials to accurately isolate and quantify micro-charcoal $(<150 \mu \mathrm{m})$ fragments (Earle et al. 1996; Horn et al. 1992). Today, access to greater computing power, higher resolution digital images, and a wider array of image analysis software has made automated and digital charcoal analysis increasingly common (Crawford and Belcher 2014; Springer et al. 2012). Scientists using these methods indicate that they can be faster than visual methods (Whitlock and Larsen 2001), can measure multiple metrics (Table 1), such as fragment size class, aspect ratio, area, or volume (Ali et al. 2009), and may be more accurate in quantifying micro-charcoal particles $(<200 \mu \mathrm{m})$ in some contexts (Halsall et al. 2018).

But the benefits of digital, automated image analysis should be considered within the context of its limitations. Automated analyses commonly rely on a single greyscale threshold value to determine the presence and shape of dark colored charcoal fragments within a sample (Thevenon and Anselmetti 2007). However, removing an analyst's discretion and expertise to single out charcoal fragments can lead automated methods to misidentify dark or opaque non-charcoal particles as charcoal (Whitlock and Larsen 2001). Additionally, these methods may also underestimate charcoal fragment area or shape due to lighting conditions that lead to detection of darker colors within the center of charcoal fragments scaling to lighter colors at the edges. These processes may result in the non-detection of small fragments or misdetection of the edges of larger fragments (Hawthorne and Mitchell 2016). Unfortunately, the use of proprietary software or unpublished opensource software configurations poses challenges in improving these existing digital methods and analytical workflows. 
Table 1 Descriptions of metrics collected by CharTool in the charcoal fragment quantification and morphotype recording module. Description adapted from ImageJ User Guide (Schneider et al. 2012).

\begin{tabular}{|c|c|}
\hline Metric & Description \\
\hline Area & Area of selected pixels in calibrated units \\
\hline Min & Minimum greyscale value \\
\hline Max & Maximum greyscale value \\
\hline Perimeter & Length of outside boundary of selected pixels in calibrated units \\
\hline $\mathrm{BX}$ & X-coordinate of upper left corner of the smallest rectangle enclosing the selected pixels \\
\hline BY & Y-coordinate of upper left corner of the smallest rectangle enclosing the selected pixels \\
\hline Width & Width of smallest rectangle enclosing the selected pixels \\
\hline Height & Height of smallest rectangle enclosing the selected pixels \\
\hline Major & Length of the primary axis of the best fitting ellipse enclosing the selected pixels \\
\hline Minor & Length of the secondary axis of the best fitting ellipse enclosing the selected pixels \\
\hline Angle & Angle (0-180 degrees) of the primary axis of the best fitting ellipse enclosing the selected pixels \\
\hline Circularity & $\begin{array}{l}\text { Calculated as } 4 \pi \times \text { area } \div \text { perimeter }{ }^{2} \text {; A value of } 1.0 \text { indicates the selected pixels are a perfect circle; } \\
\text { As values approach } 0.0 \text {, the selected pixels are increasingly elongated }\end{array}$ \\
\hline Feret & $\begin{array}{l}\text { Feret's diameter of the selected pixels, which is the longest distance between any two points along } \\
\text { the selection boundary }\end{array}$ \\
\hline FeretX & Starting X-coordinate of the selected pixels Feret's diameter \\
\hline FeretY & Starting Y-coordinate of the selected pixels Feret's diameter \\
\hline FeretAngle & $\begin{array}{l}\text { Angle ( } 0-180 \text { degrees) of the Feret's diameter of the selected pixels to a line parallel to the } x \text {-axis of } \\
\text { the image }\end{array}$ \\
\hline MinFeret & Minimum caliper diameter of the selected pixels \\
\hline Aspect Ratio & $\begin{array}{l}\text { Aspect ratio of selected pixels; calculated as the major (primary) axis / minor (secondary) axis of the } \\
\text { best fitting ellipse }\end{array}$ \\
\hline Round & Roundness of selected pixels; calculated as $4 \times$ area $\div\left(\pi \times\right.$ major (primary) axis $\left.{ }^{2}\right)$ \\
\hline Solidity & Solidity of selected pixels; calculated as area/convex area \\
\hline Rectangularity & $\begin{array}{l}\text { Calculated as Perimeter } \div((\text { Width }+ \text { Height }) \times 2) \text {; A value of } 1.0 \text { indicates the selected pixels are a } \\
\text { perfect rectangle; As values approach } 0.0 \text {, the selected pixels are increasingly circular; As values } \\
\text { surpass } 1.0 \text {, the selected pixels are increasingly irregular in shape }\end{array}$ \\
\hline
\end{tabular}

Open Science and Digital Data in Archeology, Paleoecology, and Paleo-Fire Sciences

Open science is not, strictly speaking, a series of methods, standards, or a publication style. Rather, open science is a fundamental change in the culture of creating knowledge that opens the process to a diverse and emergent community of stakeholders, each with their own values, priorities, and relationships to each other and the public (Bartling and Friesike 2014). This multidisciplinary discourse has coalesced around several central themes: 1) open tools for scientific collaboration, 2) open data for comparison and replication, and 3) open access to datasets, results, and the knowledge-creation process (Faniel et al. 2018; Marwick 2017; Williams et al. 2018b). Scholars within the multidisciplinary paleoenvironmental science community, including archaeology, paleoecology, and paleo-fire science, have critically engaged with these themes, adapting them to the specific challenges associated with the incompleteness of our datasets, the often-destructive nature of our data collection, and inter-dataset comparisons or syntheses needed to track long-term global environmental change (Faniel et al. 2018; 
Williams et al. 2018a). As the community moves toward a culture of open science, we must consider the specific imperatives for digitized and born-digital data, together with the tools, methods, and analyses we apply to them.

Open data creation and digital data curation present multiple opportunities for the paleoenvironmental science community to eliminate much of the opacity surrounding digital datasets and amplify their ability to be shared, re-used, and reanalyzed. Open data creation and curation emphasize broader access to analytical tools and methods, in addition to the data they generate, through the use of free and opensourced software, scripted analyses (such as analytical sequences written in $\mathrm{R}$ or Python), and data repositories. Not only does this approach provide greater equity in access to scientific tools and data for institutions around the globe without the resource capacity for equipment or technological expenditures, but it encourages greater research reproducibility and accountability (Marwick 2017). These efforts are already underway in multiple disciplines; data repositories are currently curating and sharing data in archaeology (e.g., tDAR [McManamon et al. 2017]; Open Context [Kansa et al. 2020]; Archaeology Data Service [Wright and Richards 2018]), paleoecology/ palynology (e.g., Neotoma Paleoecology Database [Williams et al. 2018b]; European Pollen Database [Fyfe et al. 2009]); and paleo-fire science (e.g., Global Paleofire Database [Aleman et al. 2018]). These repositories represent substantial efforts toward data standardization and metadata creation that greatly amplify the potential for long-term data interpretability and re-usability (Marwick 2017), in addition to providing opportunities for large-scale synthesis (Hantson et al. 2016).

Unfortunately, many of the published methods and workflows for both visual and digital charcoal analysis do not align with the strategies for open data creation and data curation. A review of published charcoal analyses using digital methods reveals that most studies utilize 1) closed-source or proprietary software (Crawford and Belcher 2014; Horn et al. 1992; Thevenon and Anselmetti 2007); 2) opensource software without accessible code for customized features (Springer et al. 2012; Umbanhowar Jr. and McGrath 1998); 3) limited descriptions of how image analysis or other automated methods were used to quantify charcoal (Halsall et al. 2018; Hawthorne and Mitchell 2016); additionally, 4) no studies included access to curated digital data or scripted analyses via supplemental information or a digital repository. To meet the challenges of open science in charcoal analysis, current digital methods should be adapted to employ open-source software, replicable workflows, and integration with digital repositories for streamlining the transition from digital data creation and data curation.

\section{Introduction to CharTool and Descriptions of Modules}

The CharTool suite of tools for ImageJ addresses these limitations by providing a standardized, digital workflow for quantifying charcoal fragments and sediment properties, scripted modules for transparency and customization, and data preparation for upload into a digital data repository. CharTool operates as a macro extension for ImageJ and can be customized using ImageJ's scripted macro language (see ImageJ developer information; Rasband 2018). Image $\mathrm{J}$ is a freely available, open-source program used for scientific image analysis. Originally introduced in 1987 as a simple yet powerful program for biological imaging on personal computers, ImageJ has thrived thanks to continued hosting of the project by the National Institutes of Health (NIH) and an openaccess research community interested in adapting and developing the software for multidisciplinary applications (as reviewed in Schneider et al. 2012). Image J is currently used in digital charcoal analysis (e.g., Chrzazvez et al. 2014; Halsall et al. 2018; Hawthorne and Mitchell 2016; Springer et al. 2012); however, these published methods using ImageJ still do not provide adequate descriptions of the image parameters, plugins, or software version used, thus making replication and comparability difficult.

CharTool is composed of three modules for measuring charcoal and describing their associated sedimentary contexts. The modules include: 1) the charcoal fragment quantification and morphotype recording module, 2) the sediment color assessment module, and 3) the expedient sediment grain size estimation module. Functionally, CharTool occupies a unique position between traditional visual methods and digital, image-based methods for measuring charcoal fragments and sediments by using strategies from both approaches. For example, the charcoal quantification module relies on an analyst's discretion in selecting charcoal fragments to be quantified before digitally measuring 21 metrics for each selected 
fragment (Table 1). The sediment quantification module also requires an analyst's input to select areas of interest to summarize color values or sediment grain sizes from unprocessed or reserved sediments associated with charcoal samples. Consequently, CharTool is best described as a digitally-enhanced visual charcoal analysis workflow with outputs that are comparable to both visual selection and digital measurement methods.

CharTool is designed for use with a USBconnected digital microscope (with video output) or microscope eyepiece mounted microscope video camera. Video microscope output enables an analyst to deploy a live video feed into ImageJ, allowing CharTool modules and analyses to be applied to a live display that is adjustable in real time. CharTool is currently written for ImageJ version $1.52 \mathrm{k}$ but is backward and forward compatible with other versions of ImageJ on MacOS, Windows, and Linux operating systems. A description of each CharTool module is presented below. Each module requires an analyst to enter metadata for each analyzed sample (e.g., sample number, analyst name, and depth interval), which is included when a CharTool result table is exported. All tables are exported in a comma-separated values (.csv) file format. Finally, a detailed digital workflow, a stepby-step user guide for installation and use of CharTool in ImageJ, and R scripts for compiling, displaying, and uploading results to a digital repository are included as supplemental information. CharTool, its source code, and all associated materials are freely available to download ${ }^{1}$.

Charcoal Fragment Quantification and Morphotype Recording Module

This module consists of the primary tools for quantifying and classifying charcoal fragments in
CharTool and functions using the following steps: 1) charcoal fragments are visually and/or physically identified by the analyst in the view window, 2) the targeted charcoal fragment is then selected by the analyst using a semi-automated edge-finding procedure, and 3) 21 metrics are measured and recorded for the targeted charcoal fragment. This process is repeated for all charcoal fragments encountered in a sample. See Table 1 for descriptions of each metric. The user then has the option to classify each charcoal fragment into a morphotype category (Table 2). See Figure 1 for a visual sequence of this process.

Operationally, this module provides several improvements over previous charcoal analysis techniques in ImageJ. Most previous techniques rely on fixed greyscale color value thresholding to create a binary classification of charcoal (darkest pixels) and non-charcoal (lighter colored pixels) within the image (e.g., Crawford and Belcher 2014; Halsall et al. 2018; Hawthorne and Mitchell 2016; Springer et al. 2012). Greyscale values are substantially influenced by lighting conditions, variation in charcoal morphology, and the color of underlying sediment or organic material, which can lead to high rates of misclassification in automated procedures (Whitlock and Larsen 2001). For these reasons, CharTool does not use an automated procedure. Instead, CharTool requires an analysis to first identify a charcoal fragment through the Image J viewing window using standard visual methods. Upon clicking the fragment, CharTool creates a selection around its border using an edgefinding procedure that identifies all connected pixels of similar greyscale value (Figure 1). Edge-finding tolerances are adjustable, in real time, for each fragment, meaning they can be altered based on

Table $\mathbf{2}$ CharTool morphotype category based on the classifications described in Enache and Cumming (2006).

\begin{tabular}{|c|c|c|c|}
\hline Shape & Modifier & Type & Description \\
\hline \multirow[t]{4}{*}{ Geometric } & Compact & Type C & $\begin{array}{l}\text { Fragments that are compact and angular in shape. No structure or wall porosity } \\
\text { is visible. }\end{array}$ \\
\hline & & Type S/B & $\begin{array}{l}\text { Fragment that are compact and angular in shape. Structure or wall porosity is } \\
\text { visible, and the charcoal fragment is either fully black (Type S) or partially black } \\
\text { (Type B). }\end{array}$ \\
\hline & Elongated & Type D & Elongated, branch-like fragments with ramifications or spikes. \\
\hline & & Type F & Elongated, branch-like fragments without ramifications or spikes. \\
\hline \multirow[t]{2}{*}{ Irregular } & & Type M & Fragments that are irregular in shape with highly irregular porosity throughout. \\
\hline & & Type P & Fragments that are irregular in shape without any visible interior structure. \\
\hline
\end{tabular}



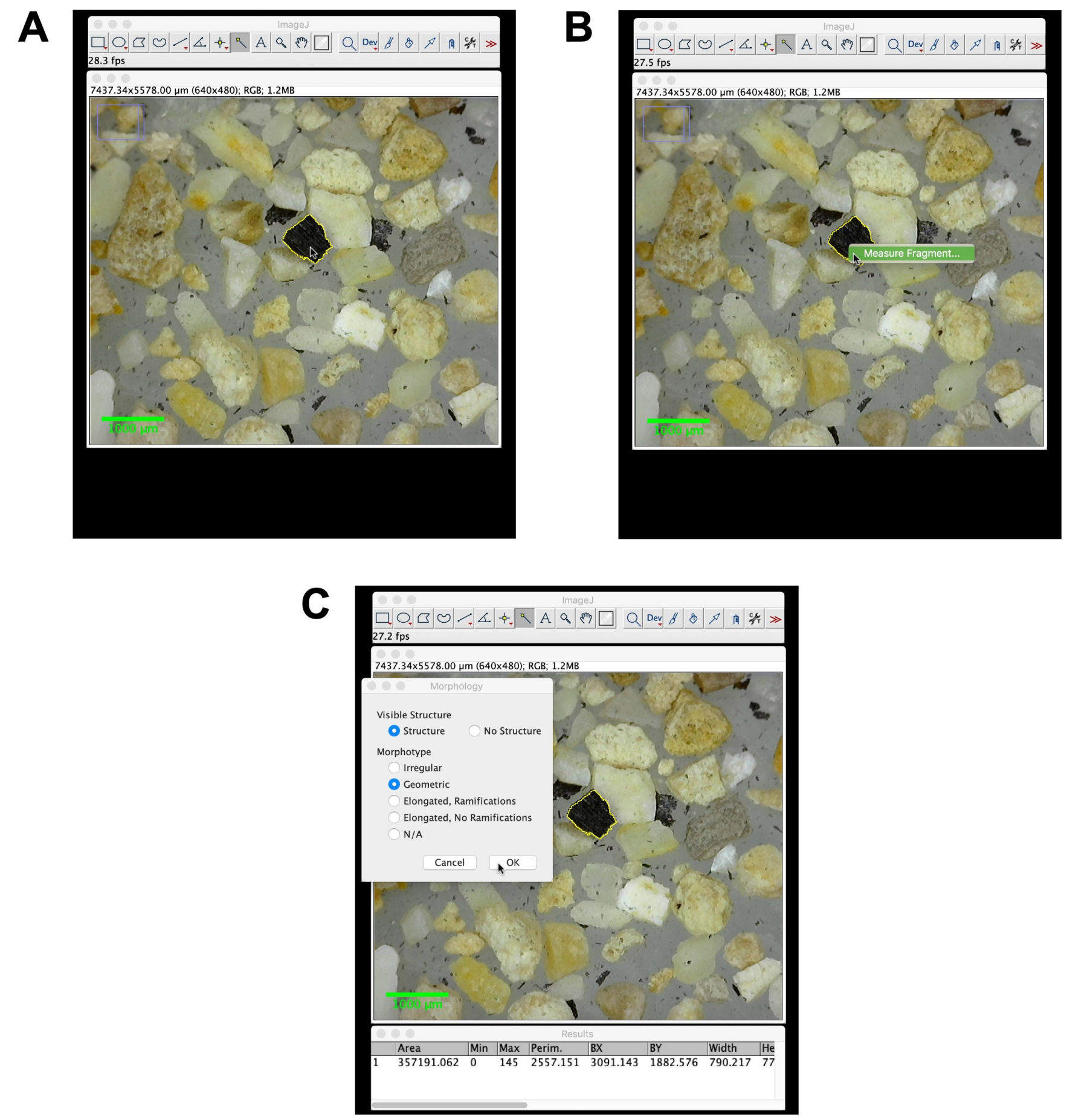

Figure 1 Overview of charcoal fragment quantification and morphotype recording module workflow in CharTool. A The analyst selects a particle they have identified as a charcoal fragment by clicking on it within the ImageJ viewing window. B After making any necessary adjustments to the selection, the analyst right-clicks to measure the fragment. C ImageJ then measures 21 metrics and initiates the morphotype selection window. After a morphotype category is selected, the morphotype is added to that fragment's entry in the results table. 
lighting conditions and charcoal color. This method decreases the likelihood edges or other light-colored portions of the fragment are excluded and provides an opportunity for the analyst to manual check the selection's accuracy.

Unlike other applications of ImageJ in charcoal analysis which use still images (i.e., Springer et al. 2012), CharTool allows an analyst greater flexibility in identifying and quantifying charcoal fragments by applying thresholds, edge-finding, and calculating measurements through a live microscope video-feed. Manipulating or repositioning the sample using a live video feed allows for adjustability in the edge-finding threshold values based on a charcoal fragment's relation to background material and assists in identifying charcoal fragments that might not be recognizable from a single view or angle. If a charcoal fragment cannot be easily selected from the background material through a series of physical manipulations or changes in threshold values, the user has the option to manually trace the charcoal fragment using the freehand selection operation available through a keyboard shortcut in CharTool.

Finally, Char'Tool incorporates an optional charcoal fragment morphotype classification component for each charcoal fragment that is selected by the user. Several studies have suggested that combustion conditions and fuels consumed during a fire determine the morphology and structure of charred fragments (Courtney Mustaphi and Pisaric 2014; Enache and Cumming 2006; Jensen et al. 2007; Walsh et al. 2018). Thus, charcoal fragment morphology is increasing used as a metric for estimating fuel sources and intensities of landscape fires. After the analyst encounters, selects, and measures a charcoal fragment, the morphotype classification workflow automatically opens a graphical window with a decision tree for classifying the fragment into a morphotype category. CharTool currently uses the morphotype classification system developed by Enache and Cumming (2006), although users can update the classification system by augmenting the current categories or adding additional morphotypes to suit the needs of their research.

\section{Sediment Color Assessment Module}

This module allows an analyst to digitally assess the color of unprocessed or reserved sediments associated with charcoal in cores, excavations, or profile sampling. Sediment color is related to mineral composition, organic matter content, or other sediment properties; when interpreted alongside charcoal metrics, sediment color can aid in evaluating depositional properties related to fire activity (NRCS Soil Survey Staff 1999). Sediment color is often evaluated in the field or in the laboratory using visual comparison to a color reference collection (i.e., the Munsell Soil Color Chart). The digital procedure in CharTool offers an additional method for assigning color values by detected color values of each pixel in the image, thus enabling color descriptions to be part of a digital workflow.

The sediment color module in Char'Tool measures the mean red, blue, and green (RBG) color values for a selected area of the digital image within the current ImageJ viewing window. The module first individually evaluates the red, blue, and green color channels in each pixel before aggregating these values into a final mean RBG value. This process limits the influence of small variations in color or individual sediment particles, clasts, or inclusions, and diminishes the effect of particle shadows on the overall color values. The RBG output can easily be converted to hue, value, and chroma attributes within the Munsell Soil Color system if desired for a particular analysis or visualization (see $\mathrm{R}$ scripts in supplemental material for an example using the munsell package).

\section{Expedient Sediment Grain Size Estimation Module}

Grain size is also an important sediment property used to evaluate depositional processes and charcoal preservation (Roos 2015). Common pedological laboratory procedures for sediment grain size include dry sediment sieving, hydrometer measurement, and laser particle size analysis to determine the texture or proportional percentages of clay, silt, and sand grains within a soil, sediment, or lithozone (NRCS Soil Survey Staff 1999). A growing number of paleo-fire studies are focused on alluvial sequences to evaluate watershed scale changes in fire history (see Frechette and Meyer 2009; Roos 2015 for examples); in these contexts coarse-grained sediment fractions, such coarse sands, gravels, and pebbles, are indicators of high velocity deposition and erosion associated with fire activity in the watershed. In an effort to meet the needs of alluvial applications of charcoal and sediment analysis, the CharTool sediment grain size evaluation module adapts ImageJ's particle size analysis workflow to estimate average grain sizes for sand particles (63 $\mu \mathrm{m})$ or larger. The module allows the user to digitally 


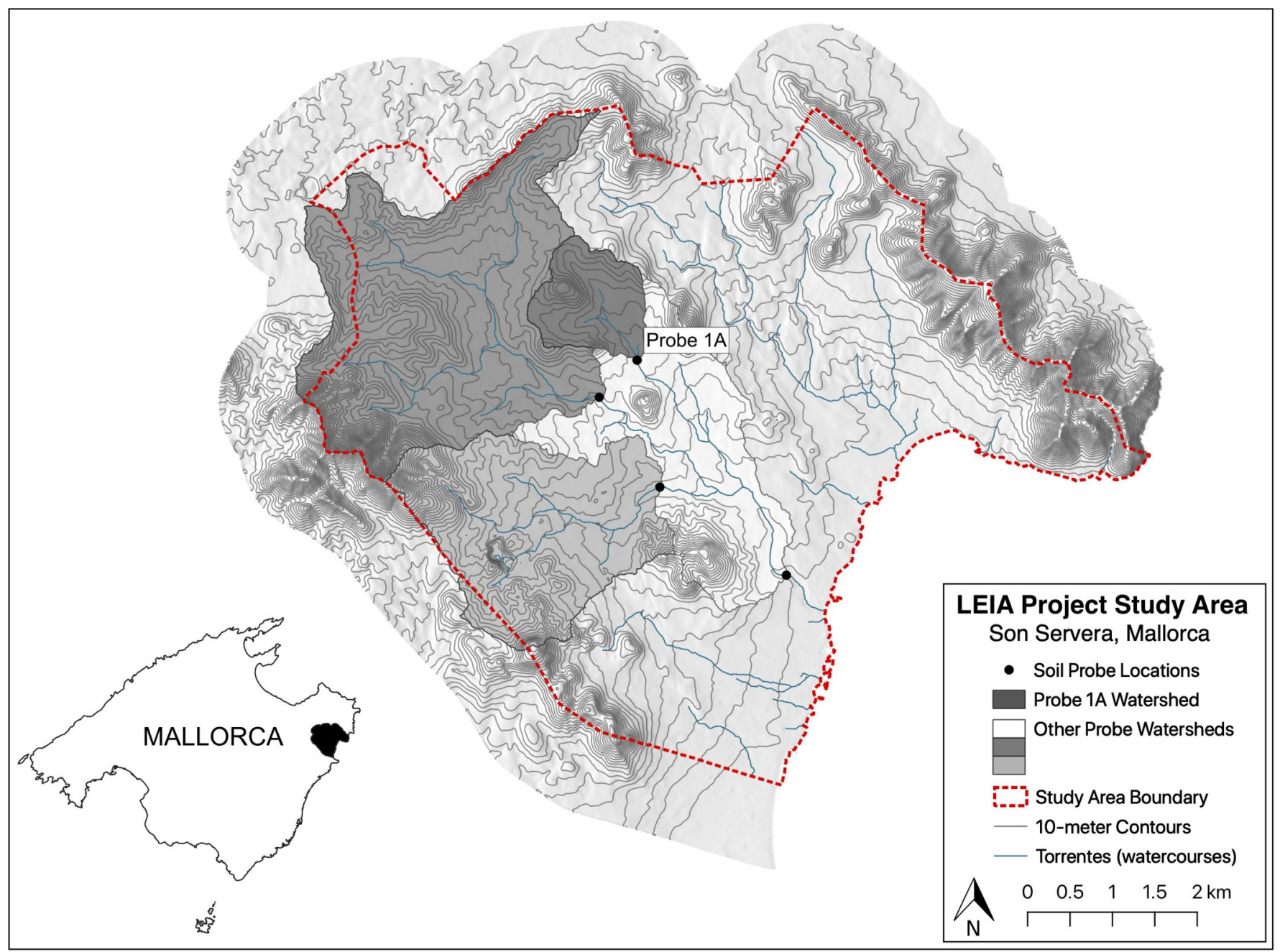

Figure 2 Sampling locations and watersheds within the LEIA Son Servera Study Area, Mallorca, Spain. Probe 1A is indicated in the center of the study area.

adjust lighting conditions and color thresholds to identify as many particles as possible within the current field of view. Once a user-specified minimum number of particle size measurements are made, the module exports all of the measurements as a .csv table. Particle size summary statistics for each sample can be calculated using $\mathrm{R}$ or other statistical software.

\section{R Scripts for Visualization and Data Curation}

Finally, to facilitate replication, data standardization, and data reuse, a series of $\mathrm{R}$ scripts are included with CharTool to allow an analyst to process CharTool outputs and visualize charcoal and sediment metrics in a vertical stratigraphic plot. An optional set of functions can be used to standardize CharTool outputs for upload to the Global Paleofire Database online data repository (International Paleofire
Network 2020). These scripts are written in $\mathrm{R}$, a free, open-source language and software for statistics and graphics, and implemented in RStudio, a free, opensource integrated development environment for $\mathrm{R}$. These scripts are well-commented and provide line-by -line guidance for all scripted analyses related to CharTool results. See the supplemental information for R, RStudio, and package versions used in these scripts.

\section{Applying Char'Tool to Sedimentary Charcoal Data: A Vignette from Son Servera, Mallorca, Spain}

As an example of how CharTool may be utilized in a research context, the following vignette outlines the integration of CharTool into sampling and laboratory processing methods common in archaeological and 

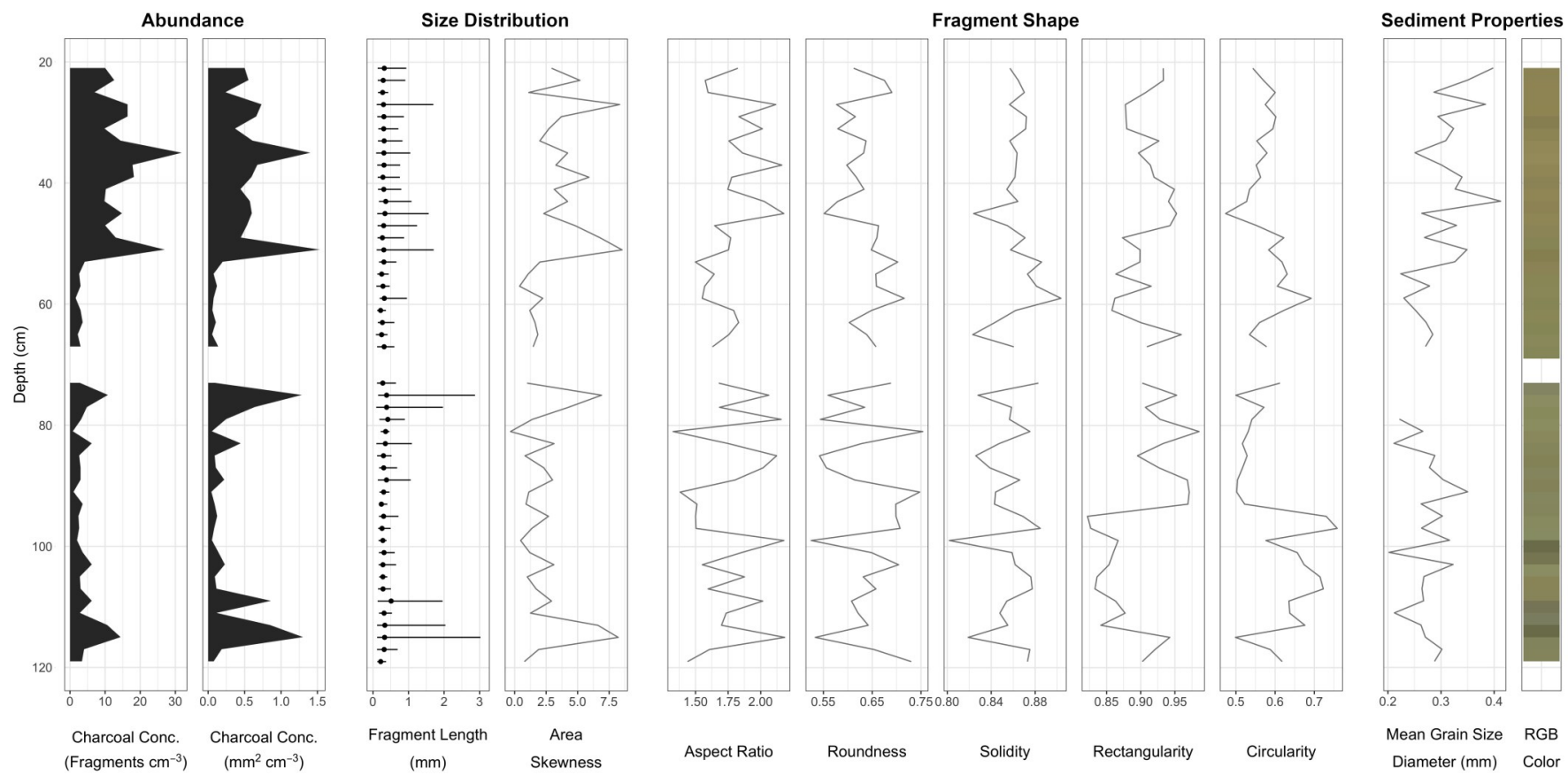

Mean Grain Size RGB

Diameter (mm) Color

Figure 3 Data from Son Servera Probe 1A generated using the charcoal fragment quantification, sediment color assessment, expedient sediment grain size estimation modules in CharTool and plotted using the ggplot 2 and cowplot packages in R. Note that samples between $68-72 \mathrm{~cm}$ could not be collected in the field and are omitted from the data below.

paleoecological charcoal analysis. The purpose of this vignette is to demonstrate the types of data that can be collected and visualized using CharTool. This example does not provide a stand-alone or comprehensive study, nor a step-by-step guide for using CharTool. For a guide to using CharTool, recommended workflow, and the scripted $\mathrm{R}$ analyses used to visualize CharTool results and prepare them for upload to a data repository, the reader is directed to the supplemental material for this article.

\section{Quantifying Charcoal to Reconstruct Fire History Related to Land-use in the Son Servera, Mallorca, Spain}

In collaboration with the Landscape, Encounters, and Identity Archaeology (LEIA) Project, an ongoing landscape archaeology field school facilitated by the University of Washington (Llobera 2019), a series of sediment probes were taken throughout watersheds within the Son Servera study area in northeast Mallorca, Spain. The objectives of LEIA Project are to use pedestrian archaeological survey, targeted excavation, and high-resolution mapping of monumental sites to understand Iron Age (c. 1100$550 \mathrm{cal}$. BCE) settlement intensification, land-use, and trade in eastern Mallorca (Llobera 2019). To complement the archaeological research, this pilot paleoecological project is aimed to assess landscapescale deposition rates within the watershed and examine spatial and temporal variation in late Holocene fire history as it relates to archaeological evidence for intensifying agricultural land-use.

Probe locations were selected using a landscapescale sampling strategy focused on alluvial sediments in nested watersheds to examine spatial and temporal variation in fire activity throughout the entire watershed (See Figure 2 for sampling locations and watersheds). Probes were placed at natural divisions/ branches in the watershed and in close proximity to areas evaluated for archaeological surface assemblages. Priority sampling areas were identified through geological maps of Holocene alluvial sediments near watercourses (or torrentes in Catalan) and were limited to areas with little evidence of recent subsurface modification or disturbance due to cultivation (i.e., trenching, terracing, or filling). Charcoal recovered from these alluvial sediments represent cumulative fire activity located upstream of the sample location. These materials moved downstream due to channel flow, sheet wash, and other fluvial processes until they were periodically deposited. 
A $3 \mathrm{~cm}$ diameter soil push probe was used to sample sediments in $2 \mathrm{~cm}$ intervals in areas of the watershed with active deposition (e.g., bases of hillslopes and alluvial terraces). All sediments removed with the probe were photographed and described in the field. A total of four probes were collected during this study, but for brevity, only Probe 1A (Figure 2) will be discussed in this vignette.

In the lab, sediment samples were subdivided into $2 \mathrm{~cm}^{3}$ samples and evaluated for color and grain size using the sediment color assessment and expedient sediment grain size estimation modules in CharTool. Subsamples were then processed for charcoal quantification using standard protocols outlined in Whitlock and Anderson (2003). These include deflocculating and chemically lightening organics using a 3\% solution of $\mathrm{H}_{2} \mathrm{O}_{2}$ to visually isolate charcoal fragments. Samples were then wet screened through $150 \mu \mathrm{m}$ sedimentology sieves to remove the smaller sample fraction. Samples were moved to petri dishes and allowed to dry at room temperature. Once dried, the samples were analyzed using the charcoal fragment quantification module in CharTool with a Dino-Lite Pro II 1.3megapixel digital microscope at 50x magnification. The morphotype classification option was not considered appropriate for the alluvial samples collected from Son Servera (see Crawford and Belcher [2014] for an overview on the effects of alluvial transport on charcoal morphology) and was not included in this example.

\section{Visualizing and Curating CharTool Results}

Figure 3 illustrates a subsample of charcoal metrics and sediment characteristics that can be collected using the CharTool. The goals of the LEIA Project focus on identifying changes in fire history as they relate to land-use history, so the charcoal metrics highlighted here are divided into charcoal abundance, size distribution, shape categories, and sediment properties to capture any changes charcoal assemblage or sediment attributes related to changes in fire frequency, intensity, or spatial distribution. This figure was produced using the $\mathrm{R}$ script, RStudio project file, and associated packages. These are provided in the supplemental material, along with the original data collected using Char'Tool, for the reader to use to replicate Figure 3.

To ensure that these data will be accessible and comparable to other charcoal datasets, they are standardized and formatted for upload to the Global Paleofire Database (GPFD) using an R script included in the supplemental material. The GPFD is an online, digital data repository that provides public access to charcoal and other paleo-fire datasets. The repository aims to promote data standardization to facilitate greater data re-use for global and regional syntheses of fire activity and model validation (Aleman et al. 2018). The database requires a user to specify metadata regarding the sampling location, context, and publications, along with the dataset's sampling intervals, depths, available age-depth models, and standardized charcoal abundance measures. These data can be quickly and easily compiled and formatted using CharTool and associated R scripts.

\section{Conclusions}

The new norms of open science are highlighting the need for research practices that support open access, open data, and open methods. While quantifying charcoal fragments is a fundamental procedure in archeological, paleoecological, and paleo-fire research, current digital methods can suffer from a lack of transparency and replicability due to under-published workflows and software configurations, as well as the use of closed-source, proprietary imaging software. The Charcoal Quantification Tool (CharTool) offers one solution to these limitations by providing a suite of open-source and freely available tools for charcoal and sedimentary analysis in ImageJ. The digital approach used by CharTool draws from the strengths of both visual and digital charcoal analysis methods, while also emphasizing open data creation and curation practices specific for born-digital data. Researchers can build on CharTool by customizing it to fit their specific research needs, while also contributing to the growing community of openscience scholars pushing for new norms in our research practices.

\section{Notes}

${ }^{1}$ All of the code and software needed to run CharTool are available for download via GitHub: https:// github.com/gsnitker/CharTool; or using the following DOI: http://doi.org/10.5281/ zenodo.1434478. Additionally, a detailed workflow, user-guide, and $\mathrm{R}$ scripts for compiling, displaying, and uploading results from CharTool to a digital repository are included as supplements to this article.

\section{Acknowledgments}

I thank Marcos Llobera, Nari Miller, and the Landscape, Encounters, and Identity Archaeology (LEIA) Project for assistance in collecting the samples 
analyzed in this study. I also thank Michael Barton, Christopher Morehart, Nahil Ismail, and the other members of the Laboratory of Environmental Anthropology and Ethnobotany and the Computational Socio-ecological Laboratory at Arizona State University for their feedback while testing CharTool. Finally, I thank the three anonymous reviewers for their insightful comments.

\section{Declarations}

Permissions: None declared.

Sources of funding: This project was funded by National Science Foundation (NSF) Dissertation Improvement Grant (Award \# 1656342) and through the NSF Graduate Research Fellowship (GRFP).

Conflicts of Interest: None declared.

\section{References Cited}

Aleman, J., A. Hennebelle, B. Vannière, O. Blarquez, and the Global Paleofire Working Group. 2018. Sparking New Opportunities for Charcoal-Based Fire History Reconstructions. Fire 1:7. DOI:10.3390/fire1010007.

Ali, A. A., P. E. Higuera, Y. Bergeron, and C. Carcaillet. 2009. Comparing Fire-History Interpretations Based on Area, Number and Estimated Volume of Macroscopic Charcoal in Lake Sediments. Quaternary Research 72:462-468. DOI:10.1016/j.yqres.2009.07.002.

Bartling, S., and S. Friesike, eds. 2014. Opening Science. Springer International Publishing, Cham. DOI:10.1007/978-3-319-00026-8.

Bliege Bird, R., D. W. Bird, L. E. Fernandez, N. Taylor, W. Taylor, and D. Nimmo. 2018. Aboriginal Burning Promotes Fine-Scale Pyrodiversity and Native Predators in Australia's Western Desert. Biological Conservation 219:110-118. DOI:10.1016/ j.biocon.2018.01.008.

Bowman, D. M. J. S., J. Balch, P. Artaxo, W. J. Bond, M. A. Cochrane, C. M. D'Antonio, R. DeFries, F. H. Johnston, J. E. Keeley, M. A. Krawchuk, C. A. Kull, M. Mack, M. A. Moritz, S. Pyne, C. I. Roos, A. C. Scott, N. S. Sodhi, T. W. Swetnam, and R. Whittaker. 2011. The Human Dimension of Fire Regimes on Earth. Journal of Biogeography 38:22232236. DOI:10.1111/j.1365-2699.2011.02595.x.

Chrzazvez, J., I. Théry-Parisot, G. Fiorucci, J.-F. Terral, and B. Thibaut. 2014. Impact of Post-
Depositional Processes on Charcoal Fragmentation and Archaeobotanical Implications: Experimental Approach Combining Charcoal Analysis and Biomechanics. Journal of Archaeological Science 44:3042. DOI:10.1016/j.jas.2014.01.006.

Courtney Mustaphi, C. J., and M. F. J. Pisaric. 2014. A Classification for Macroscopic Charcoal Morphologies Found in Holocene Lacustrine Sediments. Progress in Physical Geography 38:734-754. DOI:10.1177/0309133314548886.

Crawford, A. J., and C. M. Belcher. 2014. Charcoal Morphometry for Paleoecological Analysis: The Effects of Fuel Type and Transportation on Morphological Parameters. Applications in Plant Sciences 2:1400004. DOI:10.3732/apps.1400004.

Earle, C. J., L. B. Brubaker, and P. M. Anderson. 1996. Charcoal in Northcentral Alaskan Lake Sediments: Relationships to Fire and LateQuaternary Vegetation History. Review of Palaeobotany and Palynology 92:83-95. DOI:10.1016/0034-6667 (95)00095-X.

Enache, M. D., and B. F. Cumming. 2006. Tracking Recorded Fires Using Charcoal Morphology from the Sedimentary Sequence of Prosser Lake, British Columbia (Canada). Quaternary Research 65:282-292. DOI:10.1016/j.yqres.2005.09.003.

Faniel, I. M., A. Austin, E. Kansa, S. W. Kansa, P. France, J. Jacobs, R. Boytner, and E. Yakel. 2018. Beyond the Archive: Bridging Data Creation and Reuse in Archaeology. Advances in Archaeological Practice 6:105-116. DOI:10.1017/aap.2018.2.

Frechette, J. D., and G. A. Meyer. 2009. Holocene Fire-Related Alluvial-Fan Deposition and Climate in Ponderosa Pine and Mixed-Conifer Forests, Sacramento Mountains, New Mexico, USA. The Holocene 19:639-651. DOI:10.1177/0959683609104031.

Fyfe, R. M., J.-L. de Beaulieu, H. Binney, R. H. W. Bradshaw, S. Brewer, A. Le Flao, W. Finsinger, M.J. Gaillard, T. Giesecke, G. Gil-Romera, E. C. Grimm, B. Huntley, P. Kunes, N. Kühl, M. Leydet, A. F. Lotter, P. E. Tarasov, and S. Tonkov. 2009. The European Pollen Database: Past Efforts and Current Activities. Vegetation History and Archaeobotany 18:417-424. DOI:10.1007/s00334-009 -0215-9. 
Halsall, K. M., V. M. Ellingsen, J. Asplund, R. H. W. Bradshaw, and M. Ohlson. 2018. Fossil Charcoal Quantification Using Manual and Image Analysis Approaches. The Holocene 28:1345-1353. DOI:10.1177/0959683618771488.

Hantson, S., S. Kloster, M. Coughlan, A.-L. Daniau, B. Vannière, T. Brücher, N. Kehrwald, and B. I. Magi. 2016. Fire in the Earth System: Bridging Data and Modeling Research. Bulletin of the American Meteorological Society 97:1069-1072. DOI:10.1175/ BAMS-D-15-00319.1.

Hawthorne, D., and F. J. G. Mitchell. 2016. Identifying Past Fire Regimes throughout the Holocene in Ireland Using New and Established Methods of Charcoal Analysis. Quaternary Science Reviews 137:45-53. DOI:10.1016/ j.quascirev.2016.01.027.

Horn, S. P., R. D. Horn, and R. Byrne. 1992. An Automated Charcoal Scanner for Paleoecological Studies. Palynology 16:7-12. DOI:10.1080/01916122.1992.9989403.

International Paleofire Network. 2020. Global Paleofire Database [web page]. Available at: https://paleofire.org/. Accessed on June 19, 2020.

Jensen, K., E. A. Lynch, R. Calcote, and S. C. Hotchkiss. 2007. Interpretation of Charcoal Morphotypes in Sediments from Ferry Lake, Wisconsin, USA: Do Different Plant Fuel Sources Produce Distinctive Charcoal Morphotypes? The Holocene 17:907-915.

DOI:10.1177/0959683607082405.

Kansa, S. W., L. Atici, E. C. Kansa, and R. H. Meadow. 2020. Archaeological Analysis in the Information Age: Guidelines for Maximizing the Reach, Comprehensiveness, and Longevity of Data. Advances in Archaeological Practice 8:40-52. DOI:10.1017/aap.2019.36.

Llobera, M. 2019. University of Washington's Landscape, Encounters, and Identity Project Field School [web page]. Available at: http:// leiap.weebly.com/. Accessed September 1, 2019.

Marwick, B. 2017. Computational Reproducibility in Archaeological Research: Basic Principles and a Case Study of Their Implementation. Journal of Archaeological Method and Theory 24:424-450. DOI:10.1007/s10816-015-9272-9.
Marwick, B., J. d'Alpoim Guedes, C. M. Barton, L. A. Bates, M. Baxter, A. Bevan, E. A. Bollwerk, R. K. Bocinsky, T. Brughmans, A. K. Carter, C. Conrad, D. A. Contreras, S. Costa, E. R. Crema, A. Daggett, B. Davies, B. L. Drake, T. S. Dye, P. France, R. Fullagar, S. Graham, M. D. Harris, J. Hawks, S. Heath, D. Huffer, E. C. Kansa, S. W. Kansa, M. E. Madsen, J. Melcher, J. Negre, D. Fraser, R. Opitz, D. C. Orton, P. Przystupa, M. Raviele, J. RielSalvatore, P. Riris, I. Romanowska, N. Strupler, I. I. Ullah, H. G. Van Vlack, N. VanValkenburgh, E. C. Webster, J. Wells, J. Winters, and C. D. Wren. 2017. Open Science in Archaeology. $S A A$ Archaeological Record 17:8-14.

McManamon, F. P., K. W. Kintigh, L. A. Ellison, and A. Brin. 2017. tDAR: A Cultural Heritage Archive for Twenty-First-Century Public Outreach, Research, and Resource Management. Advances in Archaeological Practice 5:238-249. DOI:10.1017/ aap.2017.18.

Nelle, O., V. Robin, and B. Talon. 2013. Pedoanthracology: Analysing Soil Charcoal to Study Holocene Palaeoenvironments. Quaternary International 289:1-4. DOI:10.1016/j.quaint.2012.11.024.

NRCS Soil Survey Staff. 1999. Soil Taxonomy: A Basic System of Soil Classification for Making and Interpreting Soil Surveys, 2nd edition. United States Department of Agriculture, Natural Resources Conservation Service, Washington, DC.

Rasband, W. S. 2018. Image] [web page]. U. S. National Institutes of Health, Bethesda, Maryland. Available at: https://imagej.nih.gov/ij/. Accessed on June 19, 2020.

Roos, C. I. 2015. Western Apache Pyrogenic Placemaking in the Mountains of Eastern Arizona. In Engineering Mountain Landscapes: An Anthropology of Social Investment, edited by L. L. Scheiber and M. N. Zedeño, pp. 116-125. University of Utah Press, Salt Lake City, UT.

Schlachter, K. J., and S. P. Horn. 2010. Sample Preparation Methods and Replicability in Macroscopic Charcoal Analysis. Journal of Paleolimnology 44:701-708. DOI:10.1007/s10933-009 -9305-z.

Schneider, C. A., W. S. Rasband, and K. W. Eliceiri. 2012. NIH Image to ImageJ: 25 Years of Image 
Analysis. Nature Methods 9:671-675. DOI:10.1038/ nmeth.2089.

Scott, A. C. 2010. Charcoal Recognition, Taphonomy and Uses in Palaeoenvironmental Analysis. Palaeogeography, Palaeoclimatology, Palaeoecology 291:1139. DOI:10.1016/j.palaeo.2009.12.012.

Springer, G. S., L. Nivanthi Mihindukulasooriya, D. Matthew White, and H. D. Rowe. 2012. MicroCharcoal Abundances in Stream Sediments from Buckeye Creek Cave, West Virginia, USA. Journal of Cave and Karst Studies 74:58-64. DOI:10.4311/2010AN0148R1.

Thevenon, F., and F. S. Anselmetti. 2007. Charcoal and Fly-Ash Particles from Lake Lucerne Sediments (Central Switzerland) Characterized by Image Analysis: Anthropologic, Stratigraphic and Environmental Implications. Quaternary Science Reviews 26:2631-2643. DOI:10.1016/ j.quascirev.2007.05.007.

Umbanhowar Jr., C. E., and M. J. McGrath. 1998. Experimental Production and Analysis of Microscopic Charcoal from Wood, Leaves and Grasses. The Holocene 8:341-346. DOI:10.1191/095968398666496051.

Walsh, M. K., H. J. Duke, and K. C. Haydon. 2018. Toward a Better Understanding of Climate and Human Impacts on Late Holocene Fire Regimes in the Pacific Northwest, USA. Progress in Physical Geography 42:478-512. DOI:10.1177/0309133318783144.

Whitlock, C., and R. S. Anderson. 2003. Fire History Reconstructions Based on Sediment Records from Lakes and Wetlands. In Fire and Climatic Change in Temperate Ecosystems of the Western Americas, edited by T. T. Veblen, W. L. Baker, G. Montenegro, and T. W. Swetnam, pp. 3-31. Springer, New York. DOI:10.1007/0-387-21710-X_1.
Whitlock, C., and C. Larsen. 2001. Charcoal as a Fire Proxy. In Tracking Environmental Change Using Lake Sediments: Vol 3 Terrestrial, Algal, and Siliceous Indicators, edited by J. P. Smol, H. J. B. Birks, W. M. Last, R. S. Bradley, and K. Alverson, pp. 75-97. Springer Netherlands, Dordrecht, Netherlands. DOI:10.1007/0-306-47668-1_5.

Williams, J. W., E. C. Grimm, J. L. Blois, D. F. Charles, E. B. Davis, S. J. Goring, R. W. Graham, A. J. Smith, M. Anderson, J. Arroyo-Cabrales, A. C. Ashworth, J. L. Betancourt, B. W. Bills, R. K. Booth, P. I. Buckland, B. B. Curry, T. Giesecke, S. T. Jackson, C. Latorre, J. Nichols, T. Purdum, R. E. Roth, M. Stryker, and H. Takahara. 2018a. The Neotoma Paleoecology Database, a Multiproxy, International, Community-Curated Data Resource. Quaternary Research 89:156-177. DOI:10.1017/ qua.2017.105.

Williams, J. W., D. Kaufman, A. Newton, and L. von Gunten. 2018b. Building and Harnessing Open Paleodata. Past Global Change Magazine 26:49-49. DOI:10.22498/pages.26.2.49.

Wright, H., and J. D. Richards. 2018. Reflections on Collaborative Archaeology and Large-Scale Online Research Infrastructures. Journal of Field Archaeology 43:S60-S67. DOI:10.1080/00934690.2018.1511960. 